\title{
Postural abnormalities to multidirectional stance perturbations in Parkinson's disease
}

\author{
M G Carpenter, J H J Allum, F Honegger, A L Adkin, B R Bloem
}

J Neurol Neurosurg Psychiatry 2004;75:1245-1254. doi: 10.1136/jnnp.2003.021147

See end of article for authors' affiliations .....................

Correspondence to: Prof J H J Allum, University HNO-Klinik, Petersgraben 4, CH-4031 Basel, Switzerland; jallum@uhbs. ch; www.unibas. $\mathrm{ch} / \mathrm{hno} /$ neurooto

Received 19 June 2003 In revised form

12 December 2003

Accepted

14 December 2003

\begin{abstract}
Objective: We investigated trunk control, protective arm movements, and electromyographic responses to multidirectional support-surface rotations in patients with Parkinson's disease (PD), aiming to better understand the pathophysiology underlying postural instability in PD, on and off antiparkinson medication.

Methods: Ten patients with PD were compared with 11 age matched healthy controls. Seven patients were also tested without (OFF) antiparkinson medication. All subjects received rotational perturbations (7.5 deg amplitude) that were randomly delivered in six different directions.

Results: The PD patients had decreased trunk rotation and ankle torque changes, consistent with a stiffening response. Stiffness appeared to be caused by the combined action of three factors: cocontraction that interfered in particular with the normal response asymmetry in trunk muscles; increased response amplitudes in agonist and antagonist muscles at both medium ( $\sim 80 \mathrm{~ms})$ and balance correcting ( $120 \mathrm{~ms}$ ) response latencies; and increased background activity in lower leg, hip, and trunk muscles. Although the patients had significantly earlier onset of deltoid muscle responses, this gave no functional protection because the arm movements were abnormally directed. Most instability in PD occurred for backward falls, with or without a roll component. Medication provided partial improvement in arm responses and trunk roll instability.

Conclusions: Our results confirm previous findings in ankle muscles, and provide new information on balance impairments in hip, trunk, and arm responses in PD.
\end{abstract}

$\mathrm{B}$ alance impairment and falls are important features of Parkinson's disease (PD). ${ }^{12}$ Studies using support-surface perturbations have identified several distinguishing postural abnormalities ${ }^{13}$ :

- abnormally sized automatic postural responses, particularly enlarged "medium latency" stretch responses in lower leg muscles

- inability to modulate the response magnitude to different postural demands

- delayed initiation or reduced scaling of voluntary postural responses

- abnormal execution of compensatory stepping movements.

Although such postural abnormalities are clearly present during posturography experiments, many do not correlate well with clinically rated balance impairments. ${ }^{4-6}$

It is possible that an improved correlation between posturography results and clinical balance measures can be obtained using multidirectional stance perturbations. Most studies have analysed postural control in PD using a series of identical perturbations in a single direction, usually along the sagittal plane. ${ }^{5-16}$ Hence, the direction of postural perturbations may become predictable through repetition. Predictability can lead to habituation of postural responses or anticipatory strategies such as leaning, which may mask or impose postural abnormalities in PD. ${ }^{4}{ }^{17}$ Analysis of postural responses to randomly mixed perturbations in multiple directions and velocities may provide better insight into the mechanisms underlying falls in daily life because predictability would be reduced. ${ }^{18}{ }^{19}$ In addition, assessing postural responses to multidirectional perturbations could particularly help to clarify if and why there is a directional preponderance to falls in PD. ${ }^{20} 21$
A tendency for posturography studies to concentrate primarily on lower leg muscle responses ${ }^{5-10}$ may contribute to poor correlations with clinical balance measures in PD. Recent studies have confirmed the important contribution of proximal muscles to a normal balance response, particularly when perturbations are delivered in directions outside the pitch plane. ${ }^{19}{ }^{22}$ However, only a few studies have examined the effects of PD on postural reactions in more proximal muscles, ${ }^{916}$ despite mounting evidence for trunk control deficits in PD during sitting, standing, walking, or making transfers. ${ }^{23-26}$ Control of arm movements is also affected in $\mathrm{PD}$, as reflected by the reduced arm swing during walking ${ }^{24} 27$ and decreased protective arm responses to unexpected shoulder pulls. ${ }^{28} \mathrm{~A}$ similar absence of protective arm movements during falls could explain the apparently low incidence of wrist fractures in PD. ${ }^{32}$ Martin $^{30}$ showed that parkinsonian patients have abnormal trunk and arm responses to balance perturbations while seated. However, no studies have examined postural reactions of both the trunk and the arms in PD when stance was perturbed.

Therefore, the main aim of this study was to examine how changes in trunk control and protective arm movements contribute to balance deficits in PD and to determine whether common neurophysiological deficits exist in both distal and proximal muscles. The secondary aim was to determine whether postural abnormalities in PD improve with antiparkinson medication. On the basis of clinical evidence, we hypothesised that postural instability in PD would be associated with increased stiffness, altered muscular responses in both distal and proximal muscles, and absent or delayed arm movements, and that medication would provide little improvement in these postural abnormalities.

Abbreviations: $C O M$, centre of mass; ML, medium latency; PD, Parkinson's disease; UPDRSP, Unified Parkinson's Disease Rating Scale 

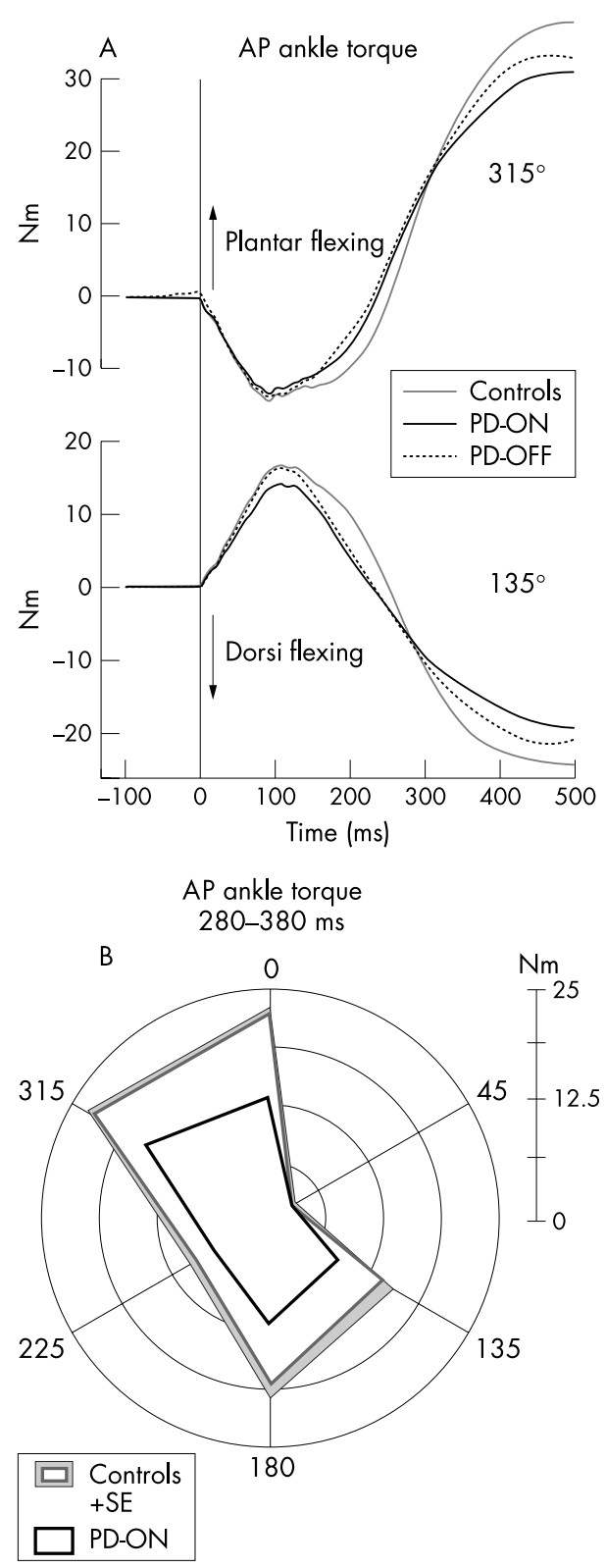

Figure 1 (A) Population sample average traces for left AP ankle torque for PD-ON, PD-OFF patients, and controls. Responses are shown for support-surface rotations of $7.5 \mathrm{deg}$ at $60 \mathrm{deg} / \mathrm{s}$, directed backward right $\left(135^{\circ}\right)$ or forward left $\left(315^{\circ}\right)$. The black vertical line at $0 \mathrm{~ms}$ represents the onset of ankle rotation. (B) Polar plots for mean absolute AP ankle torque change between 280 and $380 \mathrm{~ms}$. Each radial line (spoke) represents one of six platform directions $\left(0^{\circ}, 45^{\circ}, 135^{\circ}, 180^{\circ}\right.$, $225^{\circ}$, and $315^{\circ}$ in a clockwise notation). Note that the orientation of the radial lines does not represent the true perturbation direction. For each direction, population sample means for both PD-ON and controls and the population mean plus one standard error (SE) of the mean for controls is plotted, pooled across platform velocity. The response amplitude represented by each of the concentric circles in the plot is scaled according to the vertical scale.

\section{PATIENTS AND METHODS}

\section{Patients}

A total of 10 patients who fulfilled the UK Brain Bank criteria $^{31}$ (table 1) for idiopathic PD were included in this study. All of them sustained a clear and lasting beneficial response to levodopa or a dopamine agonist. Eleven age matched healthy elderly individuals whose responses have been described previously ${ }^{32}$ served as controls. Patients with other causes of balance impairment, a Mini Mental State
Table 1 Baseline clinical characteristics*

\begin{tabular}{llll}
\hline & $\begin{array}{l}\text { Patients } \\
(\mathbf{n}=10)\end{array}$ & $\begin{array}{l}\text { Controls } \\
(\mathbf{n}=11)\end{array}$ & p value \\
\hline Age (years) & $63.7(6.1)$ & $68.1(4.8)$ & $\mathrm{NS}$ \\
No. of women (\%) & $5(50 \%)$ & $6(55 \%)$ & $\mathrm{NS}$ \\
Height (m) & $1.7(0.1)$ & $1.7(0.1)$ & $\mathrm{NS}$ \\
Weight (kg) & $70.4(12.6)$ & $76.2(13.1)$ & $\mathrm{NS}$ \\
Duration of disease (years) & $10.4(7.2)$ & - & - \\
Fallers ( 3 months) & $6(60 \%)$ & $1(9.1 \%) \dagger$ & $\mathrm{p}=0.07$ \\
Fear of falling & $6(60 \%)$ & $1(9.1 \%)$ & $\mathrm{p}=0.07$ \\
Activities-specific Balance & $62(19)$ & $89(11)$ & $\mathrm{p}<0.01$ \\
Confidence Scale (\%) & & & \\
Medication & $9(90 \%)$ & - & \\
$\quad$ Levodopa/carbidopa & $9(80 \%)$ & - & \\
Dopamine receptor agonist & $8(80 \%)$ & - & \\
Amantadine & $4(40 \%)$ & - & \\
Anticholinergic & & - & \\
Other & $4(40 \%)$ & - & \\
$\quad$ Benzodiazepine & $4(40 \%)$ & - & \\
$\quad$ Clozapine & $1(10 \%)$ & - & \\
$\quad$ Cisapride & $1(10 \%)$ & $1(9.1 \%)$ & \\
$\quad$ Omeprazole & $0(0 \%)$ & $1(9.1 \%)$ & \\
$\quad$ Diuretics & & & \\
\hline
\end{tabular}

*Data represent mean (SD), or number of persons (percentage in parenthesis).

†One control had recently fallen, due to environmental circumstances. NS, not significant.

Examination score $\leqslant 24$, considerable postural tremor or significant dyskinesias (score $>2$ on the Modified Dyskinesia Rating Scale) ${ }^{33}$ were excluded. All patients were examined during their best clinical condition ("ON"), about one hour after intake of antiparkinson medication; seven were also tested after overnight withdrawal of all antiparkinson medication ("OFF"). All patients had predictable end-ofdose wearing-off effects. The interval between start of the OFF experiments and intake of the last medication was at least 12 hours. The order of the "ON" and "OFF" experiments was counterbalanced across subjects.

Patients (both "ON" and "OFF") and controls were clinically examined using the modified Hoehn and Yahr stages, $^{34}$ the Unified Parkinson's Disease Rating Scale $\left(\right.$ UPDRS $^{34}$ and the Tinetti Mobility Index ${ }^{35}$ (table 2). All patients had bilateral signs and although some clinical asymmetry was present, an equal proportion of patients were predominantly affected on the left or right side. All subjects reported the number of falls experienced in the last

Table 2 Clinical test scores for patients both "ON" and "OFF" medication*

\begin{tabular}{|c|c|c|c|c|c|c|c|}
\hline \multirow[b]{2}{*}{ Patient } & \multirow[b]{2}{*}{ Duration } & \multicolumn{2}{|c|}{ HY score } & \multicolumn{2}{|c|}{ UPDRS motor } & \multicolumn{2}{|c|}{ Tinettił } \\
\hline & & OFF & ON & OFF & ON† & OFF & ONब \\
\hline$A$ & 23 & 4 & 2.5 & 62 & 22 & 24 & 4 \\
\hline B & 9 & $\mathrm{n} / \mathrm{a}$ & 4 & $\mathrm{n} / \mathrm{a}$ & 48 & $\mathrm{n} / \mathrm{a}$ & 16 \\
\hline C & 4 & 2.5 & 2.5 & 30 & 21 & 10 & 8 \\
\hline D & 5 & 2.5 & 2.5 & 30 & 25 & 7 & 5 \\
\hline$E$ & 9 & $n / a$ & 3 & $\mathrm{n} / \mathrm{a}$ & 50 & $\mathrm{n} / \mathrm{a}$ & 15 \\
\hline $\mathrm{F}$ & 4 & 3 & 2.5 & 50 & 36 & 8 & 5 \\
\hline G & 8 & 3 & 2.5 & 48 & 26 & 8 & 4 \\
\hline $\mathrm{H}$ & 6 & 2.5 & 2.5 & 35 & 33 & 5 & 5 \\
\hline 1 & 23 & $\mathrm{n} / \mathrm{a}$ & 3 & $\mathrm{n} / \mathrm{a}$ & 45 & $\mathrm{n} / \mathrm{a}$ & 13 \\
\hline J & 13 & 2.5 & 1.5 & 21 & 12 & 10 & 2 \\
\hline
\end{tabular}

*Medication gave a modest improvement of Unified Parkinson's Disease Rating Scale (UPDRS) motor scores and the Tinetti Mobility Index in all individual patients, except patient $\mathrm{H}$.

$\dagger$ UPDRS: ON $v$ OFF, $\mathrm{p}<0.05$.

$\ddagger$ Tinetti optimal score $=0$, worst score $=28$.

TTinetti: ON v OFF, $p=0.07$.

$\mathrm{n} / \mathrm{a}$, not available. 
three months and whether they had a fear of falling. Balance confidence was assessed using the Activities-specific Balance Confidence (ABC) scale ${ }^{36}$ (table 1).

All subjects gave witnessed informed consent according to the Declaration of Helsinki. The institutional review boards of the hospitals where subjects were outpatients (Basel and Leiden) approved the study.

\section{Measurements}

To record electromyography (EMG) signals, pairs of silversilver chloride electrodes were placed approximately $3 \mathrm{~cm}$ apart along the muscle bellies of the left tibialis anterior, the left soleus, and bilaterally on the gluteus medius muscles, the paraspinals at the L1-L2 level of the spine, and the medial deltoid muscles. EMG amplifier gains were kept constant throughout the experiments. Anterior-posterior (AP) and medio-lateral ankle torques were calculated from supportsurface reaction forces measured with strain gauges embedded in the support surface beneath each foot. ${ }^{19}$ Trunk angular velocity in the pitch (sagittal) and roll (frontal) planes was collected using Watson Industries transducers (Eau Claire, WI; $\pm 300 \mathrm{deg} / \mathrm{s}$ range) mounted to a metal plate strapped around the chest at the level of the sternum. Systron-Donner angular velocity transducers (Inglewood, CA; $\pm 200 \mathrm{deg} / \mathrm{s})$ were attached to a $10 \mathrm{~cm}$ metal plate and strapped to the lateral aspect of the left upper arm using an elasticised bandage. ${ }^{32}$ To measure the left lower leg angle in the pitch plane, a lightweight metal rod was fixed with an adjustable strap to the lateral aspect of the left tibia, about $4 \mathrm{~cm}$ below the level of the lateral condyle. The rod was connected to a potentiometer located on the pitch axis of the support surface.

\section{Procedure}

The procedures were identical with those in a previous study. ${ }^{32}$ The subject's feet were lightly strapped into heel guides on the support surface with $20 \mathrm{~cm}$ heel separation. This setup required the subject to use a foot-in-place response to recover their balance, without the option of taking a step. The ankle joint axis was aligned with the pitch axis of the rotating platform. The roll axis had the same height as the pitch axis, above the top surface of the platform, and passed equally between the feet. Just prior to the experiment, the subject was asked to assume their "preferred" standing posture with the arms hanging comfortably at their sides. At each individual's "preferred stance" position, the low pass filtered $(5 \mathrm{~Hz})$ AP torque signal was used as the reference value for the "preferred stance" for the remainder of the experiment.

The experiment consisted of two series of 44 perturbations each. The perturbations consisted of randomised combinations of six directions and two velocities (either $30 \mathrm{deg} / \mathrm{s}$ or $60 \mathrm{deg} / \mathrm{s})$, at a constant amplitude of $7.5 \mathrm{deg}$. Perturbation directions included two in the pitch plane (forward or $0^{\circ}$; and backward or $180^{\circ}$ in our notation). Combinations of pitch and roll stimuli were used to form the other four directions: forward right $\left(45^{\circ}\right)$, backward right $\left(135^{\circ}\right)$, backward left $\left(225^{\circ}\right)$ or forward left $\left(315^{\circ}\right)$. Each of the 12 different combinations of perturbation direction and velocity were randomly presented seven or eight times. Each perturbation was preceded by a random 5-20 second delay. During this period, subjects monitored an oscilloscope, which was located at eye level, approximately $1 \mathrm{~m}$ in front of them. This oscilloscope displayed the AP torque reference signal. Using this visual feedback, subjects were asked to maintain AP ankle torque within a range of $\pm 1 \mathrm{Nm}$ from their "preferred stance" reference value prior to the onset of perturbation. The perturbation commenced only when this condition was met. At the time of perturbation onset visual feedback was blanked out. Three handrails ( $80 \mathrm{~cm}$ high) were located at a distance of $40 \mathrm{~cm}$ to the sides and to the front of the platform centre and were adjustable in height. The subjects were instructed to grasp the handrails only if needed. Two assistants were present to lend support in case of a fall. To minimise fatigue, the participants were given a 2-3 minute seated rest after the 15th and 30th trial of each series, and a longer seated rest period (five minutes) between each series.

\section{Data analysis}

EMG and biomechanical recordings were initiated $100 \mathrm{~ms}$ prior to perturbation onset and had a sampling duration of one second. EMG recordings were band pass analogue filtered between $60 \mathrm{~Hz}$ and $600 \mathrm{~Hz}$, full wave rectified, and low pass filtered at $100 \mathrm{~Hz}$ using previously described filter characteristics ${ }^{37}$ prior to sampling at $1 \mathrm{kHz}$. All biomechanical data were sampled at $500 \mathrm{~Hz}$ and digitally low pass filtered off-line at $25 \mathrm{~Hz}$ using a zero phase-shift 10th order Butterworth filter. Angular velocities were integrated off-line using trapezoid numerical integration to yield angular displacement.

All recordings were averaged off-line across each perturbation direction and velocity, with the first trial of each series excluded to reduce habituation effects. ${ }^{37}$ Subject averages were then used to produce population sample averages for each single direction and velocity combination. Zero latency was defined as the first inflexion of ankle rotation velocity and did not vary with direction or subject. Ankle torque change was calculated between $280 \mathrm{~ms}$ and $380 \mathrm{~ms}$, which corresponds to the interval of largest torque change in controls. ${ }^{19}$ Average trunk angular displacement was calculated at $300 \mathrm{~ms}$ and $700 \mathrm{~ms}$ after trapezoid integration of angular velocity traces. These times were chosen because trunk pitch and roll angle peak, respectively, at these times in controls. ${ }^{32}$ Angular displacements of the arm relative to the trunk were calculated from the difference in angular velocity signals of these two body segments.

Onset latencies were analysed for directions that elicited primary balance correcting responses in each particular muscle. EMG latencies were determined individually using a semi-automatic computer algorithm that determined when the average profile exceeded two standard deviations above background muscle activity for more than $50 \mathrm{~ms}$. Each latency was first selected by the algorithm and then approved or manually corrected following inspection by one operator (MGC).

EMG areas were corrected by subtracting the average level of background activity (measured over a $100 \mathrm{~ms}$ period prior to perturbation onset) from the overall response amplitude. Corrected EMG areas were calculated using trapezoid integration within predetermined time intervals associated with stretch reflexes (40-100 ms from stimulus onset), medium latency (ML) responses (80-120 ms) and balance correcting responses (120-220 ms). ${ }^{19}$ To assess symmetry of activation between bilateral muscles, we calculated an "asymmetry index" as the ratio between left sided and right sided activity. Our primary analyses concentrated on between-groups comparison of PD-ON patients and controls using a mixed analysis of variance (ANOVA) model (group $\times$ direction) for both EMG and biomechanical data, pooled across the two stimulus velocities. Before this pooling, we checked that response scaling with velocity was not different across the populations. The skewed distributions of EMG response areas were analysed statistically following rank transformation. To determine the effect of medication on postural responses, the mixed model ANOVA was applied to repeated measures for the seven patients who were tested both ON and OFF. Significant main and interaction effects were further explored using post hoc comparisons, using 

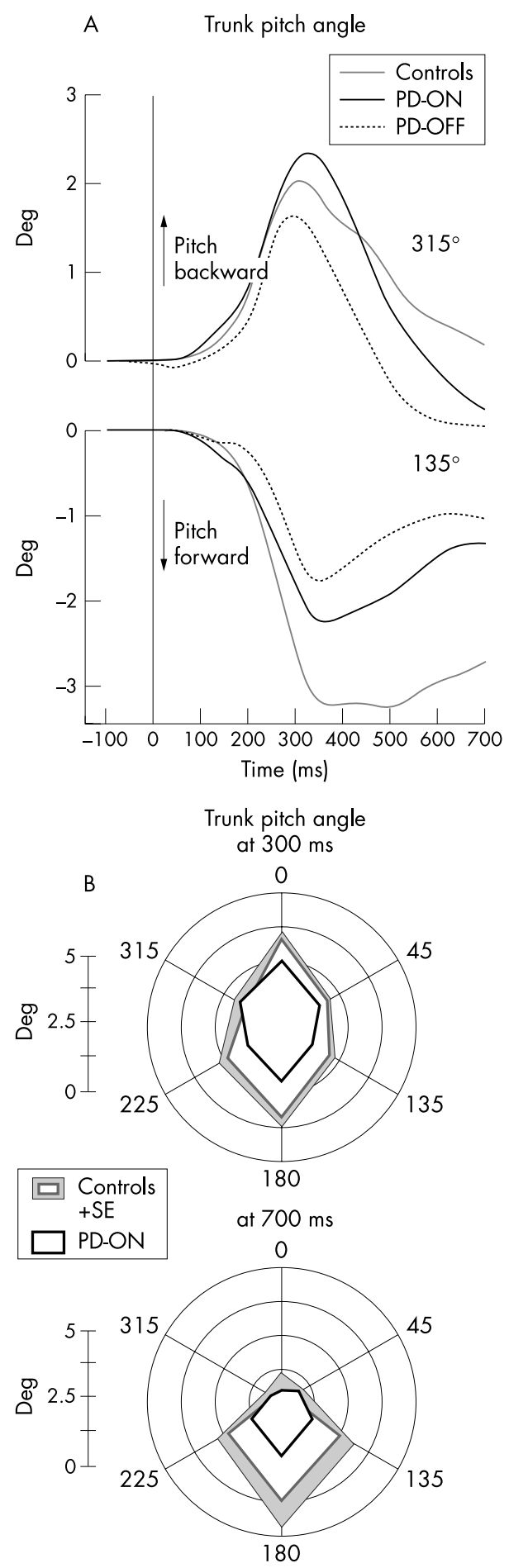

Figure 2 (A) Population average traces for trunk pitch angular displacement for PD-ON, PD-OFF patients and controls. Responses are shown for support-surface rotations of $7.5 \mathrm{deg}$ at $60 \mathrm{deg} / \mathrm{s}$, directed backward right $\left(135^{\circ}\right)$ or forward left $\left(315^{\circ}\right)$. The black vertical line at $0 \mathrm{~ms}$ represents the onset of ankle rotation. (B) Polar plots for mean absolute trunk pitch angular displacement at $300 \mathrm{~ms}$ (top plot) and $700 \mathrm{~ms}$ (bottom plot) pooled across plafform velocity. See fig 1 legend for details of the polar plots.

$t$ tests for parametric data (biomechanics and onset latencies) and non-parametric tests (Mann-Whitney and Wilcoxon's tests) for EMG amplitudes. Significance levels were set at 0.05 . As shown in the results, we found little differences in balance deficits ON and OFF medication, except for arm and trunk roll responses. Therefore, we concentrated our analysis primarily on comparisons between controls and PD-ON patients, which is consistent with the majority of previous research..$^{5-8}$ 11 $^{13-15}{ }^{38}$ Differences between PD-ON and PD-OFF patients have been reported only where changes were statistically significant.

\section{RESULTS}

\section{Biomechanical changes}

Patients and controls had similar patterns of AP torque generation, but different response amplitudes. Backward rotations of the platform caused an initial passive plantar flexing torque that increased until $\sim 150 \mathrm{~ms}$, when the AP torque reversed into an active dorsiflexion torque. Ankle torque over the first $200 \mathrm{~ms}$ did not differ between the patients and controls (fig lA). However, the torque change between $280 \mathrm{~ms}$ and $380 \mathrm{~ms}$ was significantly decreased in PD-ON patients compared with controls (fig lA, B) but not different between patients ON and OFF medication (fig lA). These group differences depended on perturbation direction (significant interaction effect of group by direction; $\left.F_{5,95}=5.55 ; \mathrm{p}<0.001\right)$. Thus, PD-ON patients had the greatest reduction in left AP ankle torque change between $280 \mathrm{~ms}$ and $380 \mathrm{~ms}$ for perturbations directed forward and to the left, with smaller differences for perturbations backward and to the right (fig 1B). Similar, but mirror image, changes were found for right ankle AP torque.

Backward perturbations caused the lower legs to pitch backwards and the trunk to pitch forwards (and vice versa for forward perturbations). Trunk pitch displacement always commenced at approximately $80 \mathrm{~ms}$ and reached a peak displacement between $300 \mathrm{~ms}$ and $350 \mathrm{~ms}$ (fig 2A). Perturbations with a roll component induced less rapid trunk roll displacement in the same direction as platform rotation, with an onset of approximately $150 \mathrm{~ms}$, and a final peak angular displacement at 500-600 ms.

When trunk pitch displacements were measured at $300 \mathrm{~ms}$ and $700 \mathrm{~ms}$ a group by direction interaction was observed between the PD-ON patients and controls $\left(F_{5,95}=2.48\right.$ and 2.67, respectively; $\mathrm{p}<0.05)$. No differences in trunk pitch were noted between PD-ON and PD-OFF patients. Differences between patients and controls were observed primarily for backward directed perturbations, where the velocity of forward trunk pitch was slower in patients, resulting in a significantly smaller peak forward displacement at $300 \mathrm{~ms}$ (fig 2A, B). These differences were consistent with decreased ankle torque in patients with PD (fig 1). There were no differences in trunk pitch displacement at $300 \mathrm{~ms}$ for forward perturbations (fig 2B). The reduction in forward trunk pitch angular displacement for backward directed perturbations was even greater at $700 \mathrm{~ms}$ (fig 2B). PD-ON patients and controls had a similar amplitude of trunk roll. However, PD-OFF patients had increased trunk roll at $700 \mathrm{~ms}$ for all perturbation directions, except for purely forward perturbations (significant interaction effect of group (ON $v$ OFF) by direction; $\left.F_{5,30}=3.13 ; \mathrm{p}<0.03\right)$.

\section{Upper arm}

Directionally dependent changes in arm responses were observed between the patients and controls. Differences were larger for patients OFF medication (fig 3A). For backward perturbations, the upper arm flexed forward reaching a peak angular displacement relative to trunk movement at $350 \mathrm{~ms}$ (fig 3A). Conversely, arm extension occurred with forward perturbations (fig 3A). Thus the arms were moved spatially ahead of the trunk. The patients had initial arm pitch responses that were similar to those of the controls in pattern and timing, but decreased in velocity and peak displacement. These differences caused a significant difference in arm 

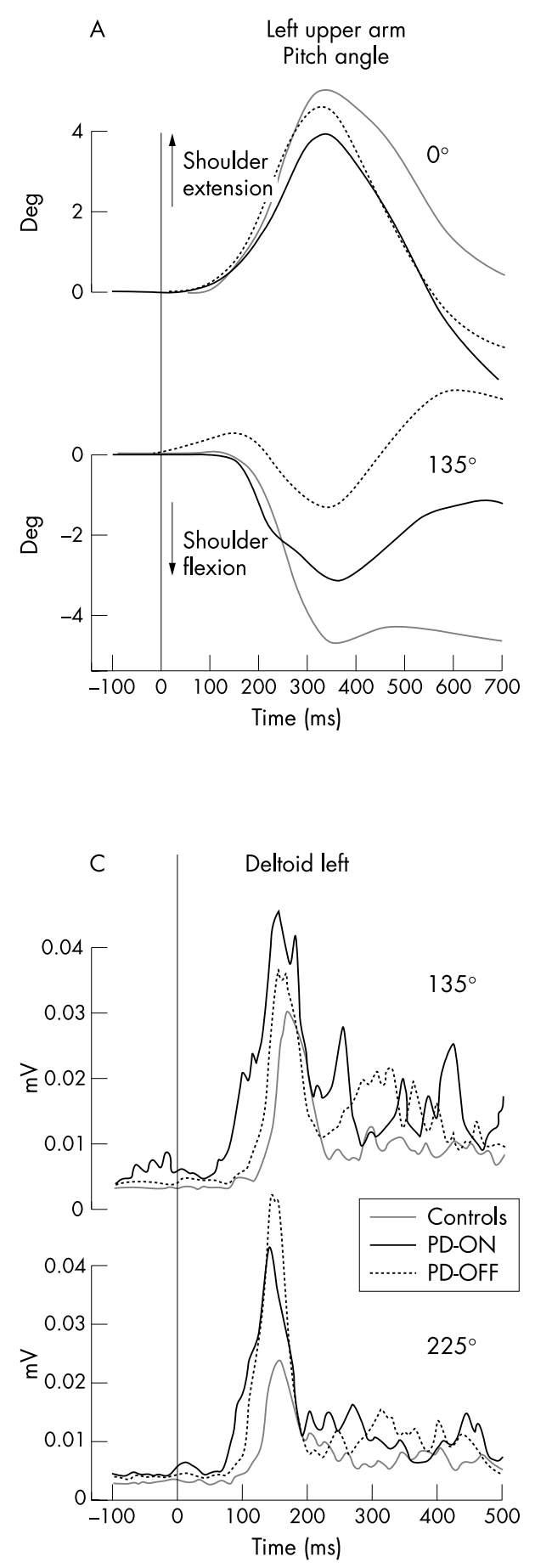
Left upper arm Pitch angle at $700 \mathrm{~ms}$
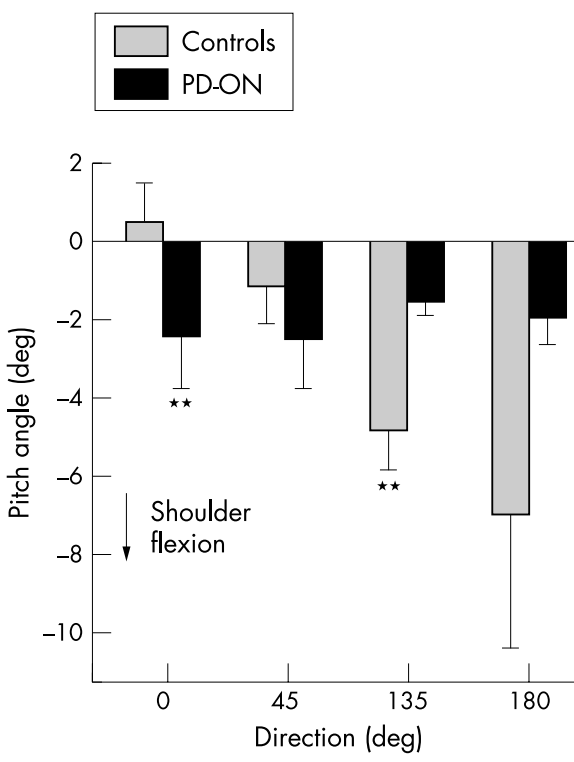

D

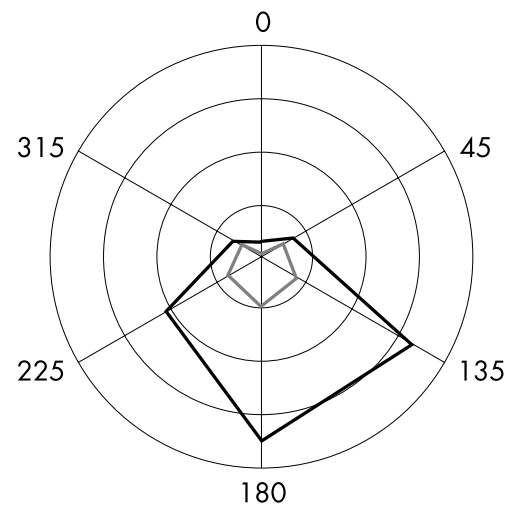

Deltoid left

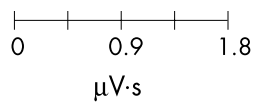

Figure 3 (A) Population sample average traces for upper arm pitch angular displacement for PD-ON, PDOFF patients, and controls. Responses are shown for support-surface rotations of $7.5 \mathrm{deg}$ at $60 \mathrm{deg} / \mathrm{s}$, directed backward right $\left(135^{\circ}\right)$ or forward $\left(0^{\circ}\right)$. The black vertical line at $0 \mathrm{~ms}$ represents the onset of ankle rotation. (B) Bar graphs include mean and standard error for upper arm pitch angular displacement at $700 \mathrm{~ms}$ pooled across velocity for forward $\left(0^{\circ}\right)$, forward right $\left(45^{\circ}\right)$, backward right

$\left(135^{\circ}\right)$, and backward $\left(180^{\circ}\right)$ directions. *Difference significant between PD-ON patients and controls $(p<0.05)$. (C) Population average traces for left medial deltoid for patients (both ON and OFF) and controls. Responses are shown for supportsurface rotations of $7.5 \mathrm{deg}$ at $60 \mathrm{deg} / \mathrm{s}$, directed, backward right $\left(135^{\circ}\right)$ or backward left $\left(225^{\circ}\right)$. (D) Polar plots for median amplitude of balance correcting responses measured between $120 \mathrm{~ms}$ and $220 \mathrm{~ms}$ for the left medial deltoid. Each radial line (spoke) represents one of six platform directions $\left(0^{\circ}, 45^{\circ}, 135^{\circ}, 180^{\circ}, 225^{\circ}\right.$, $315^{\circ}$, in a clockwise notation). For each direction, median muscle activity is plotted for PD-ON patients and controls, pooled across velocity. The response amplitude represented by each of the concentric circles in the plot is scaled according to the horizontal scale. flexion angle at $700 \mathrm{~ms}$ (interaction effect of PD-ON $v$ control by direction; $\left.F_{5,95}=3.73 ; \mathrm{p}<0.01\right)$. The controls maintained the peak arm flexion angle acquired by $350 \mathrm{~ms}$ constant, with progressively more forward flexion of the arms as perturbation directions changed from forward to backward (fig 3B). In contrast, the patients quickly reversed the upper arm movement after $350 \mathrm{~ms}$ to return the arm to a flexed position that was similar across all perturbation directions (fig 3B). They demonstrated excessive arm adduction compared with the controls. The high variability in the patients' arm movements led, however, to a lack of statistical difference in population sample means at $700 \mathrm{~ms}$. No differences between PD-ON and PD-OFF patients were observed for any arm angle measures.

\section{Muscle responses}

\section{Lower leg muscles}

Backward perturbations elicited a short latency reflex in the stretched soleus muscle at $\sim 50 \mathrm{~ms}$ followed in controls by a relatively small ML response, and a balance correcting response occurred in the tibialis anterior (fig $4 \mathrm{~A}, 225^{\circ}$ ). The latter response generated a dorsiflexion torque to counteract the backward displacement of the body. Forward perturbations first elicited a combined stretch and ML response in the tibialis anterior muscles at approximately $80 \mathrm{~ms}$, followed by a large balance correcting response in the soleus muscle (fig $4 \mathrm{~A}, 315^{\circ}$ ).

PD-ON patients had normal stretch reflex amplitudes, but ML responses were enlarged in the tibialis anterior 

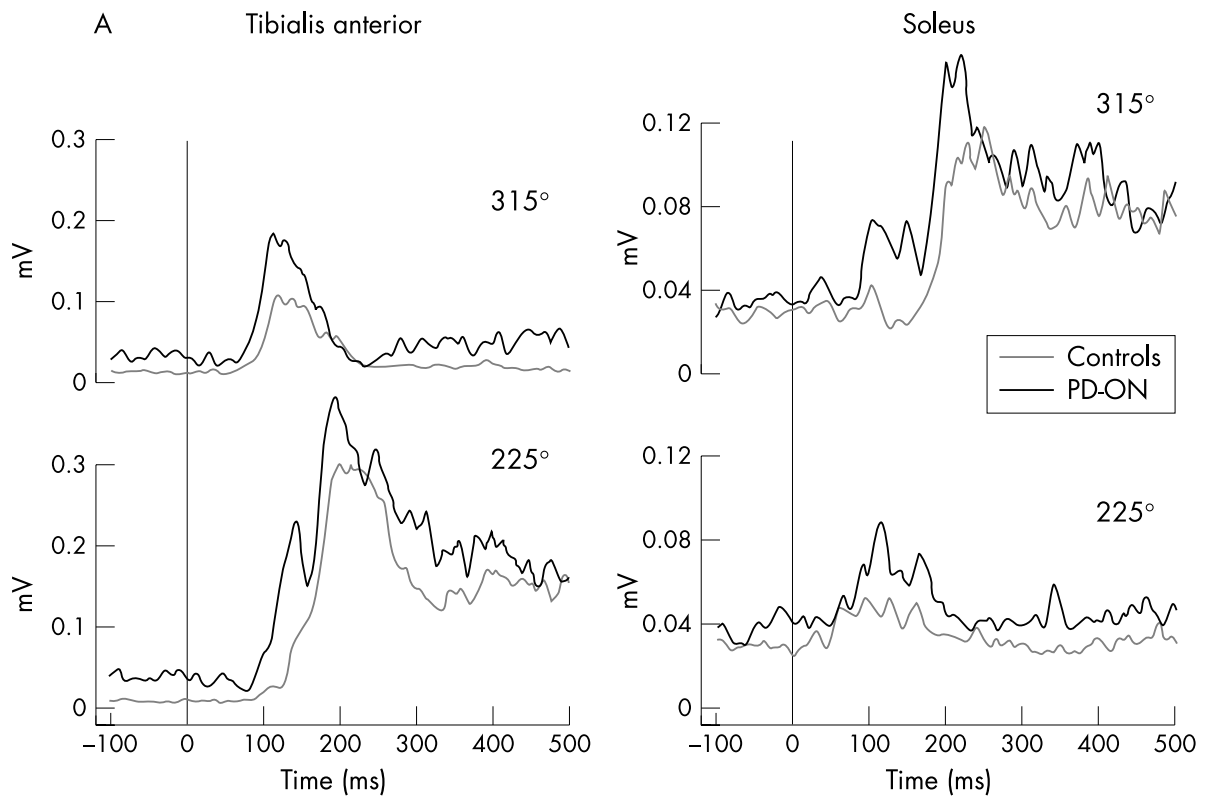

Figure 4 (A) Population sample average traces for tibialis anterior (left panel) and soleus (right panel) for patients (ON) and controls. Responses are shown for support-surface rotations of $7.5 \mathrm{deg}$ at $60 \mathrm{deg} / \mathrm{s}$, directed backward left $\left(225^{\circ}\right)$ and forward left $\left(315^{\circ}\right)$. The black vertical line at $0 \mathrm{~ms}$ represents the onset of ankle rotation. (B) Population average traces for left gluteus medius (left panel) and left paraspinal muscles (right panel) for patients (ON) and controls. Responses are shown for support-surface rotations of $7.5 \mathrm{deg}$ at $60 \mathrm{deg} / \mathrm{s}$, directed backward left $\left(225^{\circ}\right)$ and backward

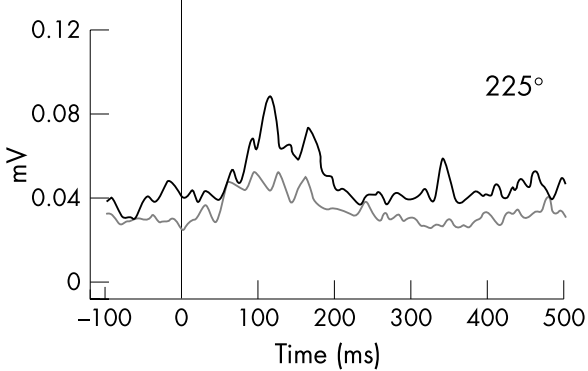
right $\left(135^{\circ}\right)$. The black vertical line at $0 \mathrm{~ms}$ represents the onset of ankle rotation.

B

Gluteus medius

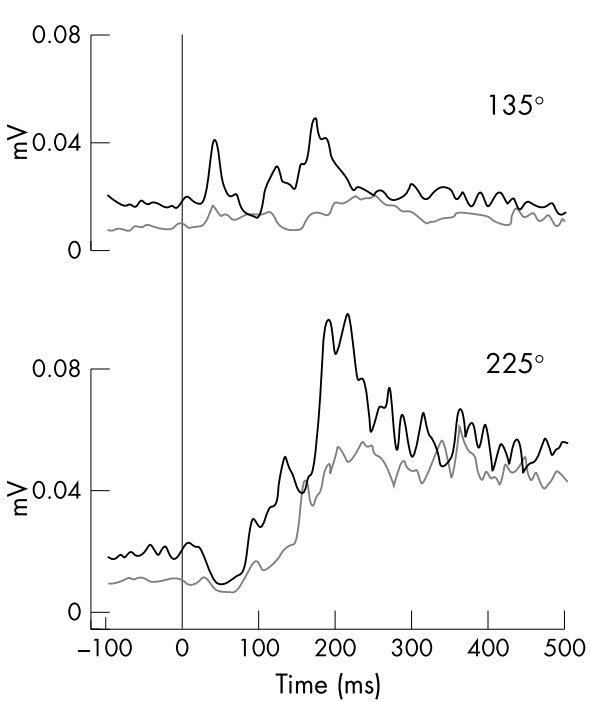

Paraspinals

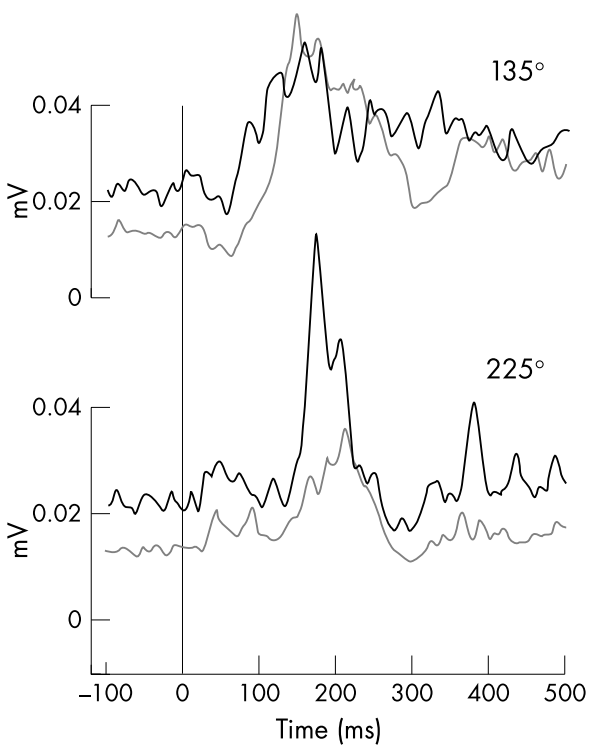

$\left(F_{1,19}=8.67 ; \mathrm{p}<0.01\right)$ and the soleus $\left(F_{1,19}=9.70 ; \mathrm{p}<0.01\right)$ independent of perturbation direction. The patients had ML responses not only for perturbation directions that stretched the muscle, but also for directions which unloaded the muscle $\left(135^{\circ}\right.$ in tibialis anterior and $315^{\circ}$ in soleus) and generated no stretch response in the controls (fig 5A). Amplitudes of balance correcting responses were also significantly influenced by PD (fig 5B). Soleus balance correcting responses were larger in $\mathrm{PD}-\mathrm{ON}$ patients compared with the controls for all perturbation directions $\left(F_{1,19}=10.01 ; \mathrm{p}<0.01\right)$. The patients also tended to have larger balance correcting responses in the tibialis anterior for backward rotations. They had higher background activity in the lower leg muscles compared with the controls, with a threefold increase in the tibialis anterior and a $25 \%$ increase in the soleus (fig 4A). Changes in amplitude of ML and balance correcting responses in the PD patients could not be explained by differences in onset latency of responses. Mean onset latencies for balance correcting responses did not differ between the PD-ON patients and controls in the tibialis anterior (for example 143.8 (SD 24.7) ms in patients $v 132.8$ (18.7) $\mathrm{ms}$ in controls for backward left perturbations), or the soleus (for example $176.9(23.0) \mathrm{ms}$ in patients $v 182.4$ (18.2) $\mathrm{ms}$ in controls for forward left perturbations).

\section{Hip and trunk muscles}

The gluteus medius and paraspinal muscles were most active for backward perturbation directions with a roll component. The largest balance correcting responses were observed in the hip and trunk muscles for perturbations that initially unloaded the muscle, that is, in the gluteus medius ipsilateral to the tilt-down of the support surface, and in the contralateral paraspinals. For example, backward left $\left(225^{\circ}\right)$ perturbations caused an initial unloading response in the left gluteus medius, followed by a large balance correcting response (fig $4 \mathrm{~B}$ ). In the controls this perturbation caused a stretch response at $40 \mathrm{~ms}$ in the left paraspinal muscle, followed by a very small balance correcting response.

Interestingly, there were few differences between the patients and controls in the proximal muscles for pure 
A

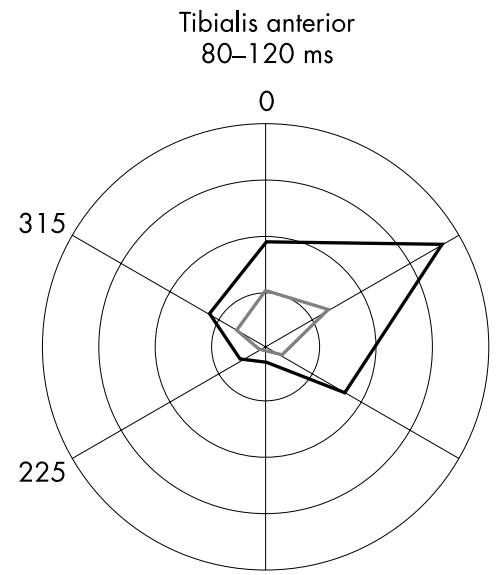

B

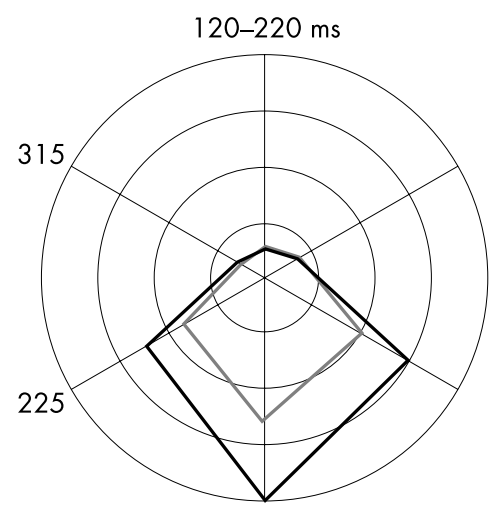

C

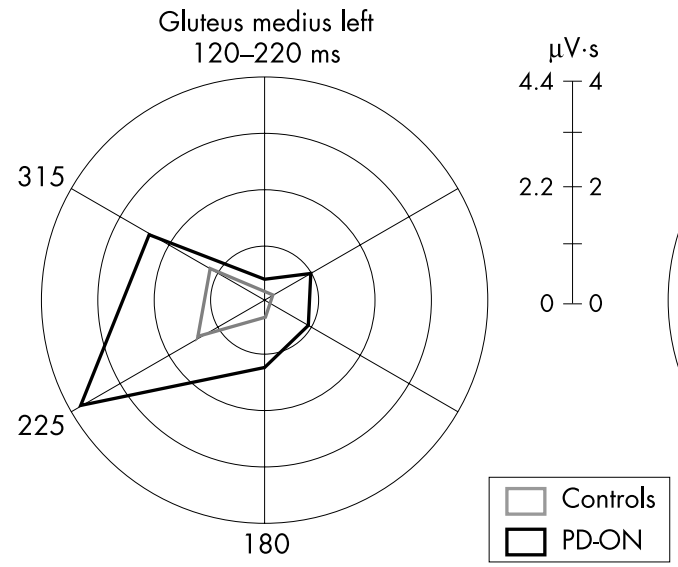

Soleus

$80-120 \mathrm{~ms}$

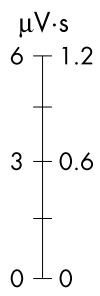

$\mu \mathrm{V} \cdot \mathrm{s}$

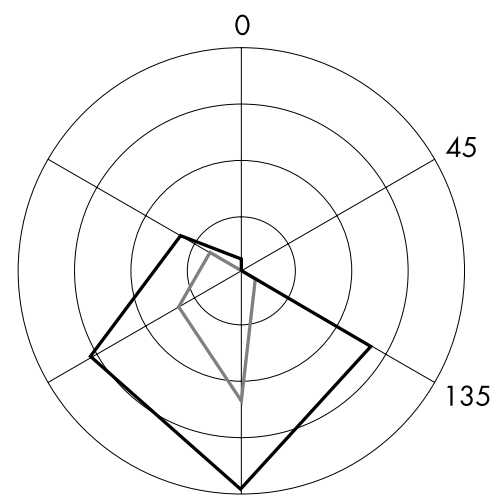

$\mu \mathrm{V} \cdot \mathrm{s}$

$32 \top^{8}$

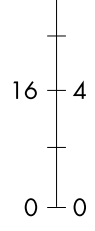

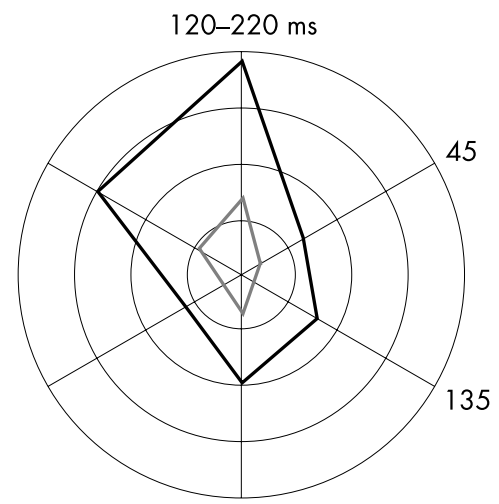

Figure 5 (A) Polar plots of median amplitude of medium latency responses measured between $80 \mathrm{~ms}$ and $120 \mathrm{~ms}$ for left tibialis anterior (left panel) and left soleus (right panel). (B) Polar plots of median amplitude of balance correcting responses measured between $120 \mathrm{~ms}$ and $220 \mathrm{~ms}$ for left tibialis anterior (left panel) and left soleus (right panel). (C) Polar plots for median amplitude of balance correcting responses measured between $120 \mathrm{~ms}$ and $220 \mathrm{~ms}$ for left gluteus medius (left plot) and left paraspinals (right plot). See fig 1B legend for details of the polar plots.

backward perturbation, and significant differences only emerged when a roll component was added to the perturbation. Amplitudes of early stretch responses in the gluteus medius did not differ between the patients and controls once these were referred to the $3 \times$ higher background activity (fig 4B). However, the patients showed bursts of ML activity at $\sim 80 \mathrm{~ms}$, in both the gluteus medius and the paraspinal muscles that were not observed in the controls, particularly for roll directions (fig 4B). Amplitudes of balance correcting responses in the gluteus medius were increased in PD-ON patients compared with controls (see polar plots of fig 5C for all perturbation directions $\left.\left(F_{1,19}=5.21 ; \mathrm{p}<0.05\right)\right)$. However, directional sensitivity was preserved in patients as reflected by analysis of the "asymmetry index" ( the ratio between left and right sided activity), which was comparable for PD-ON patients and controls (table 3 ).

The paraspinal balance correcting activity was influenced by a significant interaction effect between PD-ON versus controls and the perturbation direction $\left(F_{5,95}=4.09\right.$; $\mathrm{p}<0.01)$. The responses in the left paraspinals were significantly decreased with respect to background activity in patients for rightward perturbations which initially elicit unloading responses, and significantly increased for leftward roll perturbations which stretched the left paraspinal muscle and caused minimal activity in controls (figs 4B and 5C). The patients consequently lacked the normal left-right asymmetry in their paraspinal responses, resulting in co-contraction. This was reflected by the asymmetry ratios for backward roll 
Table 3 Asymmetry indexes for the gluteus medius and paraspinal muscles, as calculated by the ratiot between the left muscle/right muscle for each perturbation direction illustrated here for two different perturbation directions (backward left and backward right)

\begin{tabular}{llllll}
\hline & \multicolumn{2}{l}{ Gluteus medius } & & \multicolumn{2}{l}{ Paraspinals } \\
\cline { 2 - 3 } \cline { 5 - 6 } \cline { 5 - 6 } & $\begin{array}{l}\text { Backward } \\
\text { left (225) }\end{array}$ & $\begin{array}{l}\text { Backward } \\
\text { right (135) }\end{array}$ & & $\begin{array}{l}\text { Backward } \\
\text { left (225) }\end{array}$ & $\begin{array}{l}\text { Backward } \\
\text { right (135) }\end{array}$ \\
\hline Controls & 1.89 & 0.17 & & 0.18 & 3.74 \\
PD-ON & 2.64 & 0.27 & & $0.71^{*}$ & $1.54^{*}$
\end{tabular}

*Ratios significantly different from controls.

†A ratio closer to 1 represents more symmetrical activation of left and right muscles for a specific perturbation direction.

tilts (table 3), which showed asymmetrical paraspinal activation in controls, but more symmetrical activation in PD-ON patients (interaction effect between group and perturbation direction; $F_{5,95}=2.48 ; \mathrm{p}<0.05$ ).

\section{Shoulder muscles}

We observed bilateral deltoid muscle activity regardless of the perturbation direction in all subjects. The largest arm responses were observed for backward rotations, with less activity for forward roll perturbations and almost negligible activity for pure forward rotations (fig 3D). Onsets of medial deltoid responses were earlier in PD-ON patients compared with controls for purely backward perturbations (119.6 (SD 22.1) $\mathrm{ms}$ in PD-ON patients $v 140.4$ (12.3) ms in controls; $\mathrm{p}<0.05$ ) and backward right perturbations (114.3 (19.1) $\mathrm{ms} v$ 133.6 (18.2) ms, respectively; $\mathrm{p}<0.05)$. Onset latencies tended to be earlier for backward left perturbations (116.6 (28.7) $\mathrm{ms}$ in patients $v 124.1$ (18.1) $\mathrm{ms}$ in controls; $t$ test, $\mathrm{p}=0.53)$. Compared with PD-ON patients, PD-OFF patients had later onset latencies of the deltoid muscles for purely backward perturbations (133.2 (10.7) ms; p<0.05) but comparable latencies for backward right perturbations (117.5 (19.1) ms). The medial deltoid balance correcting responses tended to be larger in patients compared with controls for backward and backward roll directions (fig 3C, D), but these differences were not significant.

\section{DISCUSSION}

Our observations on multidirectional perturbations to stance revealed three distinct postural abnormalities in PD. First, patients had reduced ankle torques and decreased counterrotation of the trunk, especially when the body's centre of mass (COM) was pitched backwards suggesting that counteracting muscle torques generated active stiffening at the trunk and ankles instead of moving the COM to a more stable position. The active stiffening was caused by increased ML and balance correcting responses and co-contraction of agonists and antagonists. This effect was observed not only in the lower legs, as other studies have shown, ${ }^{810}{ }^{16}$ but also in the muscles spanning the hips and trunk. The abnormally high background activity in the patients may have contributed further to increased overall stiffness. Secondly, the patients had a significantly earlier onset of deltoid muscle responses, but this gave no functional protection because the arm movements were abnormally directed, particularly in backward directions. Thus all postural abnormalities in PD were most prominent for perturbations directed backwards, with or without a roll component. Finally, antiparkinson medication provided little to no improvement in these pitch plane abnormalities. Some improvement was noted for trunk roll.

\section{Increased trunk stiffness}

Our biomechanical analyses of pitch plane movements provide evidence for increased hip and trunk stiffness in PD. Specifically, the patients had decreased trunk pitch angle changes compared with the controls. The increased trunk stiffness in PD was presumably related to the enlarged balance correcting responses in the gluteus medius. Additional stiffness may have resulted from the loss of the normal reciprocal relationship between the stretched and unloaded paraspinals to backward and roll perturbations, as well as the increased background activity in the gluteus medius and the paraspinals. These results confirm previous clinical evidence for trunk deficits in PD. The patients have decreased range of motion of the trunk in both pitch and roll directions, ${ }^{23}{ }^{26}$ decreased trunk mobility during sit-to-stand ${ }^{28}$ and during functional reach tests, ${ }^{26}$ as well as increased axial stiffness while walking. ${ }^{24}$ Both, the elderly controls and PD patients had trunk roll displacements which were slower than those of the younger controls. ${ }^{32}$ In both of the former, the initial trunk roll (over the first $150 \mathrm{~ms}$ ) was negligible compared with the younger subjects and, when present, was mainly in the same direction as the platform perturbation, more so in PD-OFF patients. In contrast, the younger controls initially had a "hinging" response over the first $150 \mathrm{~ms}$, so the upper trunk rolled in the opposite direction to the platform roll. This helps move the COM away from the downhill side. ${ }^{19}$ Therefore, some aspects of trunk stiffness in $\mathrm{PD}$, particularly in the roll plane, may be caused by age dependent alterations in joint and intrinsic muscle stiffness and may be heightened when off medication. Indeed, we observed a similar pattern of trunk roll stiffness in an elderly woman with severe proprioceptive deficits, which was attributed to excessive background activity in the gluteus medius and the paraspinals. ${ }^{39}$ Such stiffness may help to reduce sway under static conditions, ${ }^{27}$ but can be deleterious if phasically applied to unexpected postural perturbations because the trunk action is forced to "follow" the direction of the fall.

Our findings on the stiffening effects of co-contraction could explain a historical experimental observation. When seated parkinsonian patients were tilted laterally, righting responses of the trunk seemed to be absent because the patients fell sideways in the direction of the tilt without compensating adequately. ${ }^{30}$ The present EMG recordings in the paraspinals suggest that the instability may not have been caused by a lack of postural activity in the trunk muscles (as originally suggested), but by symmetrical rather than asymmetrical bilateral activation of trunk muscles.

\section{Increased leg stiffness}

Ankle stiffness may contribute to postural instability in PD. ${ }^{810} 16$ The present study also provides evidence for increased ankle stiffness due to both increased background activity before, and increased co-contraction after the perturbation. Increased amplitudes of ML and balance correcting responses were observed in the tibialis anterior and soleus, for perturbations that either stretched or unloaded these muscles. This caused co-contraction and presumably increased ankle stiffness, rather than generating a net balance correcting torque. Thus the PD patients had a decreased ankle torque despite larger ankle muscle responses. These findings extend those of Horak et al ${ }^{16}$ who observed increased co-activation of balance correcting responses in the lower and upper legs, resulting in decreased torque production and COM displacement.

Our findings of increased stiffness, increased background activity, and abnormal co-contraction are also consistent with other neurophysiological findings in PD. For example, increased intrinsic muscle stiffness was noted in studies of mechanical muscle properties in $\mathrm{PD}^{8}{ }^{40}$ Co-contraction of 
antagonist muscles has frequently been reported in PD and may be associated with changes in reciprocal inhibition during movement. ${ }^{41}{ }^{42}$ Additional stiffness could have resulted from increased muscle activity due to a fear of falling. As observed previously, ${ }^{2}$ most of our patients were fearful and had low balance confidence scores. Through changes in cognitive set, this fear can induce compensatory stiffening due to co-contraction, just as in healthy subjects when they stand at an increased height. ${ }^{43}$

\section{Protective arm movements}

Arm movements are an important defence strategy against balance perturbations. ${ }^{44}$ Protective arm movements would be particularly vital for patients with PD to compensate for the stiffness of the trunk and ankles described above. In the present study, where we recorded bilaterally from the medial deltoid muscles, we noted some unexpected differences between the patients and controls. The controls had onset latencies in the deltoid muscles that ranged between $124 \mathrm{~ms}$ and $140 \mathrm{~ms}$ - that is, very similar to the onsets for balance correcting responses in the distal and proximal muscles. PDON patients had significantly earlier deltoid responses (range 114-119 ms) than the controls. This observation was unexpected because PD is typically characterised by akinesia. One possible explanation is that a distinct ML response was elicited in patients that blended with a normally timed, later, balance correcting response. This early response could be similar to the increased ML responses seen in the leg and trunk muscles of patients. Another explanation is that the early deltoid responses in PD represent a learned response pattern, which is triggered by a startling response. ${ }^{39}$ For example, healthy subjects can activate arm muscles earlier than normal when movement is accompanied by a startling (acoustic) stimulus. ${ }^{45}$ Startle reactions have rarely been studied for somatosensory stimuli, but if there is any resemblance to the acoustic startle reaction, then the observed onset latencies would be appropriately timed to represent such startle responses ${ }^{46}$ Our biomechanical results also revealed an abnormal control of rapid arm movements in PD. Similar to the other balance deficits, these abnormal arm movements were particularly evident for backwards displacements. For backward pitch perturbations, the controls flexed their arms forward relative to the trunk, whereas the patients reduced the amount of shoulder flexion after $300 \mathrm{~ms}$ and brought the arms closer to the trunk. The arm movements of the patients seemed rather stereotyped and lacked the normal graded pitch control for different amounts of backward pitch. Arm movements in the roll direction were also changed by PD, because the patients had increased adduction of the arms compared with controls, thereby bringing the arms closer to the trunk.

The arm flexion of controls was likely protective in nature and served to either grasp the rail or to provide an extra "counterbalance" for the body displacement backwards. Similarly, moving the arms outward after a roll perturbation normally permits a finer control of the body's COM by countering the body displacement sideways. Arm raising may also create reaction moments at the leg and trunk joints that may aid or disrupt stability, depending on the direction of arm acceleration or deceleration. ${ }^{47}$ Indeed, the pitch trunk movement tended to change around $300 \mathrm{~ms}$, suggesting that a reactive arm movement had influenced trunk movement. For the patients, the decreased arm flexion in the pitch direction and the increased adduction in the roll direction caused the arms to be neither in a position to grasp a railing for support, nor to perform its counterbalance function. This suggests that, despite early and large muscle responses, protective arm responses are poorly executed in PD. The small and abnormally directed arm responses may explain why wrist fractures seem relatively rare in PD. ${ }^{329}$ Healthy elderly persons may, however, sustain wrist fractures when they attempt to cushion a backward or lateral fall..$^{21} 32$

\section{Effects of antiparkinson medication}

Medication generally yielded little improvement of postural abnormalities in PD. Minor effects included a reduction in trunk roll and a small improvement in arm responses. There were no improvements in trunk pitch motion. These findings corroborate previous studies which found little or no improvement of postural responses with antiparkinson medication. ${ }^{10}{ }^{48}$ Even though all patients except one improved with medication, the average clinical effect of antiparkinson medication was modest (see table 2). It is possible that patients with more robust clinical responses to medication might have shown greater differences between the ON and OFF states for our posturography experiments.

\section{Impaired gain control of postural responses}

Posturography studies using pitch plane perturbations reported impaired gain control of ML responses in lower leg muscles of patients with PD. ${ }^{5-10}$ The present study extends these observations to a multidirectional environment and to different muscles. We observed a global elevation of $\mathrm{ML}$ activity in PD, not only in muscles such as the tibialis anterior that normally show stretch reflex activity over the ML period (80-120 ms) in controls, but also in muscles and for perturbation directions that normally show little ML activity. We observed enhanced ML reflexes in the soleus, in more proximal muscles such as the gluteus medius and the paraspinal muscles and, as mentioned above, possibly also in the medial deltoids.

Taken together, our findings suggest that PD is associated with a global impairment of ML gain control in any muscle, independent of stretching. Impaired ML gain control may contribute directly to balance impairment in PD, for several reasons. First, it leads to contraction in antagonist muscles just before normal balance correcting activity. Secondly, afferent information resulting from ML activity may be used to scale later balance correcting activity by acting as a "test pulse" of how the balance correction would influence body motion. ${ }^{49}$ For this reason, ML activity may be restricted to a few muscles in healthy subjects because only one test signal may be required per joint. In PD, the excessive ML responses would lead to a conflicting burden of afferent input to the central nervous system, explaining why balance correcting responses are excessive and inappropriate for many environmental demands. Indeed, enhanced balance correcting responses were observed not only in leg muscles (as observed previously), ${ }^{810}{ }^{16}$ but also in the trunk, hip, and arm muscles. The increased balance correcting responses in the gluteus medius and paraspinals were not evident for pure pitch perturbations, but only became apparent following off-pitch perturbations.

In conclusion, our findings may help to clarify some clinical features observed in PD and underscore the need for new treatment strategies.

\section{ACKNOWLEDGEMENT}

Professor A H Zwinderman is gratefully acknowledged for his expert statistical advice.

\section{Authors' affiliations}

M G Carpenter, J H J Allum, F Honegger, A L Adkin, Department of ORL, University Hospital, Basel, Switzerland

M G Carpenter, Department of Kinesiology, University of Waterloo, Canada

A L Adkin, Department of Education and Kinesiology, Brock University, Canada 
B R Bloem, Department of Neurology, University Medical Centre St Radboud, Nijmegen, the Netherlands

This project was supported by grants from the Swiss National Research Foundation (31-59'319.99) to JHJ Allum, the Natural Sciences and Engineering Research Council of Canada to MG Carpenter and AL Adkin, and the Prinses Beatrix Fonds and the Dutch Parkinson Patiënten Vereniging to BR Bloem.

Competing interests: none declared

\section{REFERENCES}

1 Rogers MW. Disorders of posture, balance, and gait in Parkinson's disease. Clin Geriatr Med 1996;12:825-45.

2 Bloem BR, Grimbergen YAM, Cramer M, et al. Prospective assessment of falls in Parkinson's disease. J Neurol 2001;248:950-8.

3 Bloem BR, van Vugt JP, Beckley DJ. Postural instability and falls in Parkinson's disease. Adv Neurol 2001;87:209-23.

4 Bloem BR, Beckley DJ, van Hilten JJ, et al. Clinimetrics of postural instability in Parkinson's disease. J Neurol 1998;245:669-73.

5 Schieppati M, Nardone A. Free and supported stance in Parkinson's disease. Brain 1991; 114:1227-44.

6 Waterston JA, Hawken MB, Tanyeri S, et al. Influence of sensory manipulation on postural control in Parkinson's disease. J Neurol Neurosurg Psychiatry 1993;56:1276-81.

7 Scholz E, Diener HC, Noth J, et al. Medium and long latency EMG responses in leg muscles: Parkinson's disease. J Neurol Neurosurg Psychiatry 1987; 50:66-70

8 Dietz V, Berger W, Horstmann GA. Posture in Parkinson's disease: impairment of reflexes and programming. Ann Neurol 1988;24:660-9.

9 Horak FB, Nutt JG, Nashner LM. Postural inflexibility in parkinsonian subjects. J Neurol Sci 1992;111:46-58

10 Bloem BR, Beckley DJ, van Dijk JG, et al. Influence of dopaminergic medication on automatic postural responses and balance impairment in Parkinson's disease. Mov Disord 1996;11:509-21.

11 Bloem BR, Beckley DJ, van Vugt JP, et al. Long latency postural reflexes are under supraspinal dopaminergic control. Mov Disord 1995;10:580-8.

12 Bloem BR, van Vugt JP, Beckley DJ, et al. Habituation of lower leg stretch reflexes in Parkinson's disease. Electroenceph Clin Neurophysiol 1998; 109:73-7.

13 Allum JH, Keshner EA, Honegger $F$, et al. Disturbance of posture in patients with Parkinson's disease. In: Amblard B, Berthoz A, Clarac F, eds. Posture and gait: development, adaptation and modulation. Amsterdam: Elsevier, 1988:245-57.

14 Beckley DJ, Bloem BR, Remler MP. Impaired scaling of long latency postural reflexes in patients with Parkinson's disease. Electroenceph Clin Neurophysiol 1993:89:22-8.

15 Chong RK, Horak FB, Frank J, et al. Sensory organization for balance: specific deficits in Alzheimer's but not in Parkinson's disease. J Gerontol A Biol Sci Med Sci 1999;54:M122-M128.

16 Horak FB, Frank JS, Nutt JG. Effects of dopamine on postural control in parkinsonian subjects: scaling, set, and tone. J Neurophysiol 1996;75:2380-96

17 Smithson F, Morris ME, lansek R. Performance on clinical tests of balance in Parkinson's disease. Phys Ther 1998;78:577-92.

18 Henry SM, Fung J, Horak FB. EMG responses to maintain stance during multidirectional surface translations. J Neurophysiol 1998:80:1939-50.

19 Carpenter MG, Allum JH, Honegger F. Directional sensitivity of stretch reflexes and balance corrections for normal subjects in the roll and pitch planes. Exp Brain Res 1999; 129:93-113.

20 Greenspan SL, Myers ER, Kiel DP, et al. Fall direction, bone mineral density, and function: risk factors for hip fracture in frail nursing home elderly. Am J Med 1998; 104:539-45.

21 Nevitt MC, Cummings SR. Type of fall and risk of hip and wrist fractures: the study of osteoporotic fractures. The Study of Osteoporotic Fractures Research Group. J Am Geriatr Soc 1993:41:1226-34.

22 Rietdyk S, Patla AE, Winter DA, et al. Balance recovery from medio-lateral perturbations of the upper body during standing. J Biomech 1999;32:1149-58.
23 Bridgewater KJ, Sharpe MH. Trunk muscle performance in early Parkinson's disease. Phys Ther 1998;78:566-76.

24 van Emmerik RE, Wagenaar RC, Winogrodzka A, et al. Identification of axial rigidity during locomotion in Parkinson disease. Arch Phys Med Rehabil 1999:80:186-91.

25 Morris ME, Huxham F, McGinley J, et al. The biomechanics and motor control of gait in Parkinson disease. Clin Biomech 2001;16:459-70.

26 Schenkman M, Clark K, Xie T, et al. Spinal movement and performance of a standing reach task in participants with and without Parkinson disease. Phys Ther $2001 ; 81: 1400-11$.

27 Nieuwboer A, de Weerdt W, Dom R, et al. A frequency and correlation analysis of motor deficits in Parkinson patients. Disabil Rehabil 1998;20:142-50.

28 Morris ME. Movement disorders in people with Parkinson disease: a model for physical therapy. Phys Ther 2000;80:578-97.

29 Bloem BR, Munneke M, Carpenter MG, et al. The impact of comorbid disease and injuries on resource use and expenditures in Parkinson's disease. Neurology 2003;61:1023-4

30 Martin JP. Tilting reactions and disorders of the basal ganglia. Brain 1965;88:855-74.

31 Hughes AJ, Daniel SE, Kilford L, et al. Accuracy of clinical diagnosis of idiopathic Parkinson's disease: a clinico-pathological study of 100 cases. $J$ Neurol Neurosurg Psychiatry 1992;55:181-4.

32 Allum JH, Carpenter MG, Honegger F, et al. Age-dependent variations in the directional sensitivity of balance corrections and compensatory arm movements in man. J Physiol (Lond) 2002;542:643-63.

33 Goetz CG, Stebbins GT, Shale HM, et al. Utility of an objective dyskinesia rating scale for Parkinson's disease: inter- and intrarater reliability assessment. Mov Disord 1994;9:390-4.

34 Lang AE. Clinical rating scales and videotape analysis. In: Koller WC, Paulson G, eds. Therapy of Parkinson's disease. New York: Marcel Dekker, 1995:21-46.

35 Tinetti ME. Performance-oriented assessment of mobility problems in elderly patients. J Am Geriatr Soc 1986;34:119-26.

36 Powell LE, Myers AM. The activities-specific balance confidence $(A B C)$ scale. J Gerontol Med Sci 1995;50A:M28-M34.

37 Keshner EA, Allum JH, Pfaltz CR. Postural coactivation and adaptation in the sway stabilizing responses of normals and patients with bilateral vestibular deficit. Exp Brain Res 1987;69:77-92.

38 Paquet N, Hui-Chan CW. Responses to dynamic head-and-body tilts are enhanced in Parkinson's disease. Can J Neurol Sci 1997:44-52.

39 Bloem BR, Allum JH, Carpenter MG, et al. Triggering of postural responses and compensatory strategies in a patient with total leg proprioceptive loss. Exp Brain Res 2002;142:91-107

40 Watts RL, Wiegner AW, Young RR. Elastic properties of muscles measured at the elbow in man: II. Patients with Parkinsonian rigidity. J Neurol Neurosurg Psychiatry 1986;49:1177-81.

41 Delwaide PJ, Pepin JL, Maertens de Noordhout A. The audiospinal reaction in Parkinsonian patients reflects functional changes in reticular nuclei. Ann Neurol 1993;33:63-9

42 Meunier S, Pol S, Houeto JL, et al. Abnormal reciprocal inhibition between antagonist muscles in Parkinson's disease. Brain 2000;123:1017-26.

43 Carpenter MG, Frank JS, Silcher CP, et al. The influence of postural threat on the control of upright stance. Exp Brain Res 2001;138:210-18.

44 Maki BE, Mcllroy WE. The role of limb movements in maintaining upright stance: the "change-in-support" strategy. Phys Ther 1997;77:488-507.

45 Carlsen AN, Hunt MA, Inglis JT, et al. Altered triggering of a prepared movement by a startling stimulus. J Neurophysiol 2003:89:1857-63.

46 Rothwell JC. Control of human voluntary movement. London: Chapman \& Hall, 1994.

47 Eng JJ, Winter DA, MacKinnon CD, et al. Interaction of reaction moments and centre of mass displacement for postural control during voluntary arm movements. Neurosci Res Commun 1992;11:73-80.

48 Bonnet AM, Loria Y, Saint-Hilaire MH, et al. Does long-term aggravation of Parkinson's disease result from nondopaminergic lesions? Neurology 1987;37:1539-42.

49 Allum JH. Responses to load disturbances in human shoulder muscles: the hypothesis that one component is a pulse test information signal. Exp Brain Res $1975 ; 22: 307-26$ 